\title{
System Identification of PWM dc-dc Converters During Abrupt Load Changes
}

\author{
Maher Algreer, Matthew Armstrong and Damian Giaouris \\ Newcastle University \\ School of Electrical, Electronic and Computer Engineering Merz Court Newcastle upon Tyne England, UK, NE1 7RU \\ Email: \{m.m.f.saber, matthew.armstrong, damian.giaouris\}@ncl.ac.uk
}

\begin{abstract}
System identification has an important role in control system design, particularly in auto-tuning and adaptive control applications. Recently, it has emerged as a significant research topic in power converter design. For this reason, this paper proposes a new on-line approach to identify the parameters of a dc-dc converter. The proposed method is capable of rapidly detecting and accounting for abrupt load changes during the transient period. In most situations the controller is blind to this change. However, the estimation algorithm is able to update the model parameters before the output reaches the peak overshoot value. As a result, the controller can minimize the effect of any parameter change. The proposed method depends on predication error variation during a rapid change of load and is designed around a simple fuzzy logic structure. An adaptive forgetting factor is used to optimize the identification process, and this varies very quickly and smoothly with the step load change. Simulation results show that the identification model matches the plant during the transient period. Importantly, the convergence rate and parameters error, the two factors use to validate the algorithm, are very good during system startup and after any abrupt load change.
\end{abstract}

Keywords- system identification, parameter estimation, dcdc converters.

\section{Introduction}

A major cause of inaccuracy in controller design is inadequate information, or poor knowledge, of the plant parameters. This is particularly a problem in power electronic converter control, due to component tolerance, ambient conditions, load changes, and aging. The issue becomes even more significant when designing a discrete domain controller due to quantization, computational delays and sampling errors. The performance of the controller design can be improved if the process information is derived directly from system experimental data [1]. This is the fundamental principle of system identification and parameter estimation. The aim of parameter estimation is to evaluate the parameters within a transfer function which has an analogous arrangement to the actual plant to be controlled [2].

Generally, system identification models can be divided into two types; nonparametric models (sometimes called direct estimation) and parametric models [2]. Nonparametric methods often use spectral analysis and correlation analysis to estimate the frequency response or impulse response of the system [3]. The actions of the system are then estimated from the frequency response without using model parametric. In the parametric technique a model structure is supposed and the parameters of the model are identified using information extracted from the system. Different approaches can be used to describe the system when using parametric techniques; for instance instrumental variable, maximum likelihood and subspace methods [3-5]. Furthermore, the parameters in the model can be identified off-line or on-line by using recursive techniques [4]. In on-line applications, real-time measured data is used to update the estimation parameters of the model on a sample by sample basis. This paper investigates an online parameter estimation technique for a dc-dc buck converter.

Recently, attention has been given to system identification of dc-dc converters. Non-parametric methods often use frequency response analysis or spectral analysis. The identification result is obtained by applying Fourier Transform methods to the cross correlation between the output of the converter and an injected, frequency rich, input signal. Typically, this is a pseudo-random binary sequence (PRBS) [6]. However, there are limitations to this type of approach. It is computationally heavy and may need to process long sequences of data. As a result, the identification process can take a significant amount of time to complete. This restricts a schemes ability to identify rapid system changes, such as abrupt load changes in dc-dc converters. An example of where this may be appropriate is in a dc-dc converter power supply for a CPU. Also, significant hardware resources may be required in terms of processing power and memory [7]. Other techniques use parametric methods. In [7], a least squares method is used to solve derivative equations for the required voltage and current signals by means of polynomial interpolation. Further computation is then required to find the system parameters. A drawback of this method is that in each sample period it requires three arrays of data; an input pulse train, output voltage, and inductor current measurements to extract the circuit components. In practical implementations, there is always a limitation to computational ability and memory size. Pitel et al [8] presents a real time parametric identification method using a form of the recursive least square method (RLS) to monitor and identify fast load changes in a switched mode dc-dc power supply application. This work accurately estimates the parameters during initial start-up of the system and during slow changes of load. However, the work concludes that it is a major challenge to estimate the load value after an abrupt change of load. This paper aims to address some of these reported issues. Specifically, it aims to make the following contributions: 
a- Drive a systematic approach to map the numerical parameters in a discrete time domain model to the equivalent circuit component values.

b- Identify abrupt load changes in dc-dc converters using a new adaptive approach based on predictive error.

c- Apply the voltage transfer function rather than current transfer function to the estimation algorithm; due to sensitivity of error change, simplicity and accuracy in identification of analytical expression.

\section{Model of Buck DC-DC Converter}

\section{A- Continuous - Time Model}

The general topology of a buck dc-dc converter is shown in Fig. 1; it includes the inductor body resistance $\left(R_{L}\right)$, and the capacitor equivalent series resistance $\left(R_{C}\right)$. The load $\left(R_{O}\right)$ is considered as part of the dc-dc converter to take account of the effect of any load change to the dynamic response of the system. It is assumed that the diode is characterized by an ideal diode. With these assumptions, it can be shown that the input duty ratio to output capacitor voltage $G_{v}(s)$ transfer function, and input duty ratio to output inductor current $G_{I}(s)$ transfer function, are described as follows [8]:

$$
\begin{gathered}
G_{V}(s)=\frac{i_{L}(s)}{d(s)}=\frac{V_{g} R_{O}}{L * C\left(R_{O}+R_{C}\right) s^{2}+\left(L+C R_{O} R_{L}+C R_{O} R_{C}+\right.} \\
\left.G_{L} R_{C}\right) s+\left(R_{O}+R_{L}\right) \\
G_{I}(s)=\frac{v_{C}(s)}{d(s)}=\frac{V_{g} C\left(R_{O}+R_{C}\right) s+V_{g}}{L C\left(R_{O}+R_{C}\right) s^{2}+\left(L+C R_{O} R_{L}+C R_{O} R_{C}+\right.} \\
\left.C R_{L} R_{C}\right) s+\left(R_{O}+R_{L}\right)
\end{gathered}
$$

The equivalent lumped parameters model can be written as:

$\left[\begin{array}{l}G_{I}(s) \\ G_{V}(s)\end{array}\right]=\left[\begin{array}{l}\frac{b_{o} s+b_{1}}{s^{2}+a_{o} s+a_{1}} \\ \frac{b_{2}}{s^{2}+a_{2} s+a_{3}}\end{array}\right]$

Where;

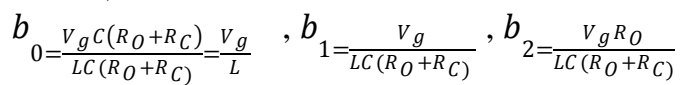

$a_{0=\frac{\left(L+C R_{O} R_{L}+C R_{O} R_{C}+C R_{L} R_{C}\right)}{L C\left(R_{O}+R_{C}\right)}}, \quad a_{1=\frac{\left(R_{O}+R_{L}\right)}{L C\left(R_{O}+R_{C}\right)}}, a_{2=} a_{0}, a_{3=} a_{1}$

Note, the current model contains two poles and one zero, whilst the voltage model exhibits two poles, but no zero.

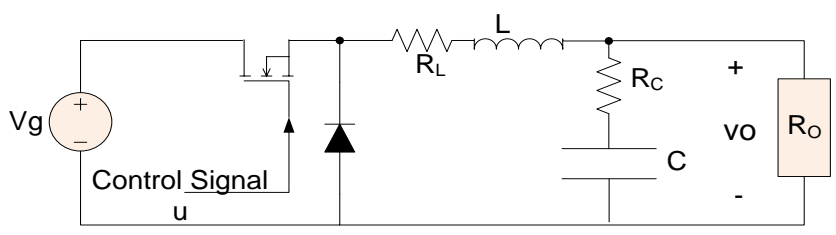

Fig. 1: General model of buck dc-dc converter

B- Discrete -Time Model

Here, a direct method of discrete parameter estimation is employed, whereby the parameters of the discrete estimation model are mapped to the general second order discrete transfer function of the buck dc-dc converter. In addition, after the mapping process is complete, this method can be used to isolate individual parameter changes, for example load variation, or in the event of a circuit component fault. The voltage transfer function for the buck dc-dc converter can be determined from (2) as follows: (a similar result may be obtained for the current model)

$G_{v}(s)=\frac{v_{c}(s)}{d(s)}=\frac{b_{2}}{(s+\alpha)^{2}+w^{2}}$

Complex pole can be described in terms of their real and imaginary parts $s=-\alpha \pm j w$, then:

$s^{\wedge} 2+a_{2} s+a_{3}=(s+\alpha+j w)(s+\alpha-j w)=$ $\left[(s+\alpha)^{2}+w^{2}\right]$

By expanding (5) and comparing coefficients with (2):

$2 \alpha=a_{2}, \quad \alpha^{2}+w^{2}=a_{3}$

In digital control systems, the zero-order hold is used almost exclusively to hold the impulse constant over a complete digital sampling period [9]. Let us say that $G_{v}(s)$ is the continuous-time transfer function of the dynamic system. Then, the discrete equivalent of $G_{v}(s)$, including the effect of the zero-order hold can be obtained by using a standard $\mathrm{s}$ to $\mathrm{z}$ domain transformation. The result is:

$G_{v}(z)=\frac{b_{2}}{\alpha^{2}+w^{2}} \frac{(A z+B)}{\left(z^{2}-2 e^{\left.-\alpha T_{S} \cos \left(w T_{S}\right) z+e^{-2 \alpha T_{s}}\right)}\right.}(7)$

Where,

$A=1-e^{-\alpha T_{s}} \cos w T_{s}-\frac{\alpha}{w} e^{-\alpha T_{s}} \sin w T_{s}$

$B=e^{-2 \alpha T_{s}}-e^{-\alpha T_{s}} \cos w T_{s}+\frac{\alpha}{w} e^{-\alpha T_{s}} \sin w T_{s}$

Here, $\mathrm{T}_{\mathrm{s}}$ is the sampling period. The discrete candidate model for the continuous system model in (1) will be:

$G(z)=\frac{Y(z)}{U(z)}=\frac{d_{1} z+d_{2}}{z^{2}+c_{1} z^{1}+c_{2}}$

Here, $\mathrm{d}_{1}, \mathrm{~d}_{2}, \mathrm{c}_{1}$ and $\mathrm{c}_{2}$ are the parameters to be identified, and are dependent on the actual circuit component values and the sampling frequency [8]. By comparing the denominators of (7) and (9), the coefficients $c_{1}$ and $c_{2}$ in (9) can be computed as:

$z^{2}+c_{1} z+c_{2}=z^{2}-2 e^{-\alpha T_{s}} \cos \left(w T_{s}\right) z+e^{-2 \alpha T_{s}}$

$c_{1}=-2 e^{-\alpha T_{s}} \cos \left(w T_{s}\right), c_{2}=e^{-2 \alpha T_{s}}$

From this,

$\alpha=\frac{\log c_{2}}{-2 T_{S}}, w=\frac{1}{T_{S}} \arccos \left(\frac{c_{1}}{-2 e^{-\alpha T_{S}}}\right)$

Using (11) the physical parameters of the dc-dc converter components can then be determined by comparing the estimated discrete-time model to the general second order discrete transfer function of the buck dc-dc converter.

It is also possible to compare the numerators of (7) and (9) to evaluate the physical parameters of the system. However, for control purposes knowledge of the pole locations is often important, and this can be directly obtained from the denominator. Also, the computation process is simpler when using the poles. 
The same procedure can be applied to the current model transfer function described in (2). It can be shown that the discrete equivalent transfer function, $G_{I}(z)$, is given by:

$G_{I}(z)=\frac{\frac{b_{0}}{w} e^{-\alpha T_{S}} \sin w T_{s}+\frac{b_{1}}{\alpha^{2}+w^{2}}(A z+B)}{\left(z^{2}-2 e^{-\alpha T_{S}} \cos \left(w T_{s}\right) z+e^{-2 \alpha T_{s}}\right)}$

Comparing (10) and (12), the denominator of the current transfer function is identical to the voltage transfer function. However, the numerator is more complex. The additional zero in the current model transfer function results in greater calculation effort, and hence computational time, to evaluate $b_{o}$. This can be seen in (12) where an additional term $\left(\frac{b_{0}}{w} e^{-\alpha T_{s}} \sin w T_{s}\right)$ is apparent. In practice, a further drawback of using the current model for identification is increased noise related to the high frequency current ripple [8]. For this reason, the voltage transfer function is the preferred model in this work.

\section{On-Line Parameter Estimation Algorithm}

\section{A- RLS Algorithm}

In real time systems, input and output data is usually processed sequentially at fixed sampling instants. In adaptive and self-tuning control systems it is essential to update the parameter estimation after each new sample becomes available. Typically, this is achieved using on line recursive techniques, which allow the designer to monitor and track parameter changes as they happen. Recursive methods are computationally efficient, making them suitable for microprocessor applications [10]. However, there is only limited literature describing the use of these methods in dc-dc power converter systems [6]. The input-output relation given in (9) may be described as a linear difference equation. Several methods exist to obtain this, however a relatively simple autoregssive- moving average (ARMA) technique is used here [11]. From this, it is possible to derive the following difference equation:

$y(k)+c_{-} 1 y(k-1)+c_{-} 2 y(k-2)=d \_1 u(k-1)+$ $d \_2 u(k-2)$

Where, $y(k)$ is the output signal, $u(k)$ is the input duty control signal, and $\left\{c_{1}, c_{2}, d_{1}, d_{2}\right\}$ are the parameters to be estimated. The classical RLS equations, including forgetting factor, are summarised as follows [11]:

$\hat{\theta}(k)=\hat{\theta}(k-1)+K(k) \varepsilon(k)$

$K(k)=\frac{P(k-1) \emptyset(k)}{\lambda+\emptyset^{T}(k) P(k-1) \emptyset(k)}$

$\varepsilon(k)=y(k)-\hat{\theta}^{T}(k-1) \emptyset(k)$

$P(k)=\frac{1}{\lambda}\left[P(k-1)-K(k) \phi^{T}(k) P(k-1)\right]$

Where, $\hat{y}(k)$ is the output estimate, $\varnothing(k)$ is the regression vector, $\hat{\theta}$ is the estimated parameter vector, $\varepsilon(k)$ is the priori error (prediction error) and $\mathrm{P}(k)$ is the covariance matrix (adaptation gain matrix). $\lambda$ is the forgetting factor $(\lambda=1$. for ordinary RLS). Initially, $P(0)=\frac{1}{\delta} I=(G I)$ (where I=Identity matrix), and $\hat{\theta}[0]=\theta_{0}$. The RLS method calculates the vector of parameter estimates by minimizing the magnitude of the prediction error. The cost function to do this is:

$J_{k}(\theta)=\frac{1}{2} \sum_{i=1}^{k}\left(y(k)-\phi^{T}(k-1) \hat{\theta}(k-1)\right)^{2}$

\section{B- Adaptive Forgetting strategy}

Using recursive estimation and adaptive techniques is an important issue where the behaviour, and hence parameters, of the system may vary over time. It is often necessary to monitor behavioural changes to optimise the controller design [9]. RLS remains an effective identification method in tracking time-varying systems. However, rapid parameters changes lead to numerical problems due to small data sets. For this reason, an appropriate choice of forgetting factor and adaption gain is vital. Generally, a small value of forgetting factor, or large adaption gain, leads to improvement in tracking ability. However, the RLS algorithm becomes very sensitive to noise. In contrast, large values of forgetting factor, or small adaption gain, results in poor tracking ability for slow parameter variations, but, the RLS algorithm is less sensitive to noise [10]. As a result, application of an adaptive forgetting factor method to a dc-dc converter system is proposed to make the identification algorithm more sensitive to change during abrupt load changes, by assigning more weight to recent samples [12].

\section{C- Fuzzy RLS Adaptive method (FRLS)}

An identification approach based on predication error is proposed. The identification structure is shown in Fig.2. A fuzzy adaptation algorithm is used to continually update the forgetting factor $(\lambda)$, based on two inputs; the squared predication error and the squared change of predication error $\left[\varepsilon^{2}(k), \Delta \varepsilon^{2}(k)\right]$. Where,

$\Delta \varepsilon^{2}(k)=\varepsilon^{2}(k)-\varepsilon^{2}(k-1)$

From which, the cost function is described as: $J_{k}(\theta)=\sum_{i=1}^{k}\left[\sum_{j=1}^{k-1} \lambda(j-i)\right]\left[\left(y(k)-\phi^{T}(k-1) \hat{\theta}(k-\right.\right.$ 1) 2

The forgetting factor adaptation mechanism is based on fuzzy rules. The membership functions are shown in Fig.3. When the predication error abruptly increases, perhaps as a result of a step change in load, $\lambda$ will quickly decrease to compensate the change by providing a large adaption gain. When the predication error is zero, representing steady state, $\lambda$ will settle to a constant value, typically approaching $\lambda=1$. However, in order to prevent the forgetting factor becoming too large, or too small, and to obtain an acceptable convergence rate at start up, a stationary rule should be added [12]. From this, the rule base shown in table I is developed. The labels are V Very Small, Small, Medium Small, Medium, 
Large, Very Large, Ultra Large\}, but for brevity are referred to as $\{V S, S, M S, M, L, V L, U L\}$.

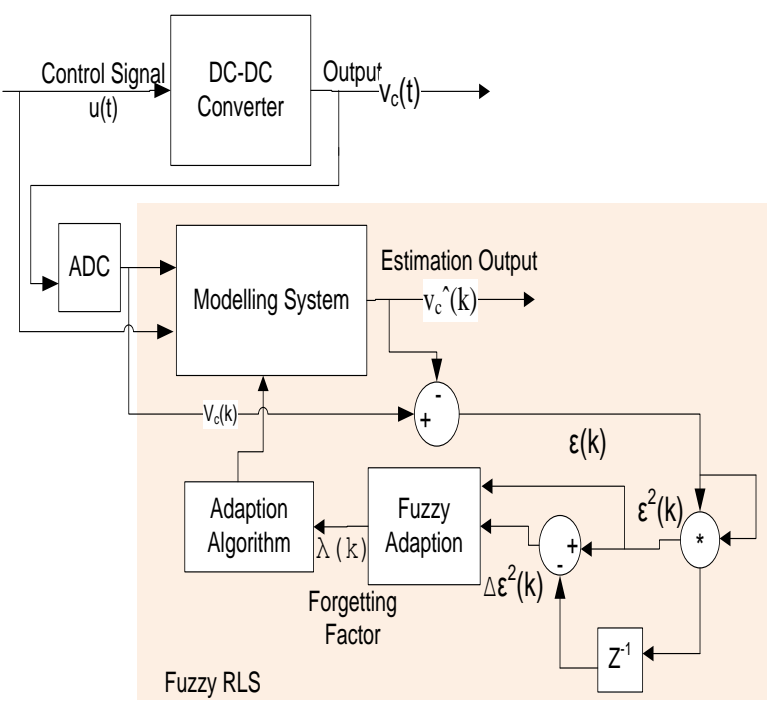

Fig. 2: System identification structure based on fuzzy RLS

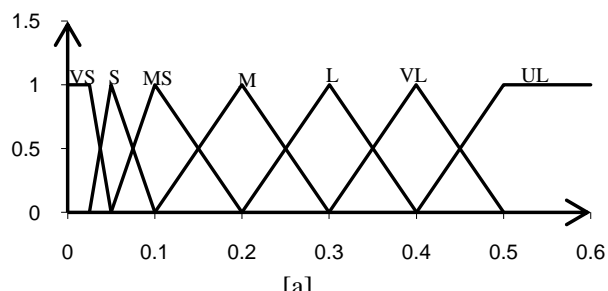

$[\mathrm{a}]$

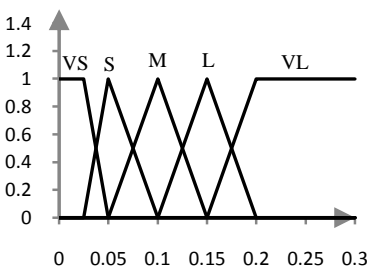

[b]

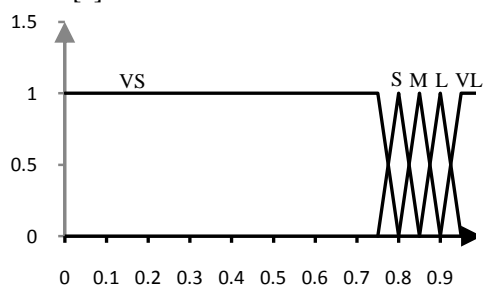

[c]

Fig.3: Membership function for input and output, a: predication error square, b: variation predication error square, c: forgetting factor

Table I: Rule base for forgetting factor $(\lambda)$

\begin{tabular}{|c|c|c|c|c|c|c|c|}
\hline$\varepsilon^{2}(\mathrm{k}) / \Delta \varepsilon^{2}(\mathrm{k})$ & $\mathrm{VS}$ & $\mathrm{S}$ & $\mathrm{MS}$ & $\mathrm{M}$ & $\mathrm{L}$ & $\mathrm{VL}$ & $\mathrm{UL}$ \\
\hline $\mathrm{VS}$ & $\mathrm{VL}$ & $\mathrm{L}$ & $\mathrm{M}$ & $\mathrm{M}$ & $\mathrm{M}$ & $\mathrm{L}$ & $\mathrm{L}$ \\
\hline $\mathrm{S}$ & $\mathrm{L}$ & $\mathrm{L}$ & $\mathrm{M}$ & $\mathrm{M}$ & $\mathrm{L}$ & $\mathrm{M}$ & $\mathrm{M}$ \\
\hline $\mathrm{M}$ & $\mathrm{L}$ & $\mathrm{M}$ & $\mathrm{M}$ & $\mathrm{M}$ & $\mathrm{L}$ & $\mathrm{M}$ & $\mathrm{M}$ \\
\hline $\mathrm{L}$ & $\mathrm{VL}$ & $\mathrm{L}$ & $\mathrm{S}$ & $\mathrm{L}$ & $\mathrm{M}$ & $\mathrm{S}$ & $\mathrm{S}$ \\
\hline $\mathrm{VL}$ & $\mathrm{VL}$ & $\mathrm{VL}$ & $\mathrm{VS}$ & $\mathrm{VL}$ & $\mathrm{M}$ & $\mathrm{S}$ & $\mathrm{VS}$ \\
\hline
\end{tabular}

\section{Results}

Convergence time, parameter accuracy, and prediction error are important metrics. These metrics determine how closely the identified model matches the actual system transfer function, and they are used to evaluate the proposed method in this paper. To evaluate the results, the test circuit of [8] is replicated. The circuit parameters of the buck converter are as follows: $R_{O}=5 \Omega, R_{L}=150 \mathrm{~m} \Omega, R_{C}=5 \mathrm{~m} \Omega$, $L=1.26 \mathrm{mH}, \quad C=1.29 \mathrm{mF}$ and $V g=10 \mathrm{~V}$. The converter is switched with $60 \mathrm{kHz}$ pulse width modulation (PWM). Inductor current and output voltage are sampled at $4 \mathrm{kHz}$, to allow sufficient time to process the identification and control algorithm. The derived transfer functions for voltage and current model are:

$G_{v}=\frac{0.1871 z+0.1828}{z^{2}-1.85 z+0.9329}$

$G_{I}=\frac{1.942 z-1.868}{z^{2}-1.85 z+0.9329}$

The dc-dc buck converter is injected with a step input, 0.5 PWM modulation depth, superimposed with $0.2 \mu \mathrm{W} / \mathrm{Hz}$ white noise at $50 \mathrm{~ms}$. At $0.2 \mathrm{~s}$ the load changes from $5 \Omega$ to $1 \Omega$. A conventional RLS algorithm is then applied to estimate the parameters of the buck converter. The result, shown in Fig.4, agrees with [8]. Here it is shown that the algorithm rapidly estimates the system parameters during the initial transient period before the output reaches its peak. This allows the controller to deal with any parameter changes and reduce the effect of the change. Lumped parameter estimation is accurate to within $\pm 2 \%$ when using the voltage model, and $\pm 5 \%$ with the current model. Importantly, the identification algorithm provides a good estimate of initial load value $\left(\mathrm{R}_{\mathrm{O}}=5 \Omega\right)$ using either voltage or current models after eight iteration cycles $(2 \mathrm{~ms})$. Unfortunately, the parameter estimation is not so accurate during an abrupt load change. This is shown in Fig.4, where there is little change in parameter estimation after the step change in load at 0.2s. This clearly demonstrates the lack of sensitivity in the conventional RLS algorithm to an abrupt load change.
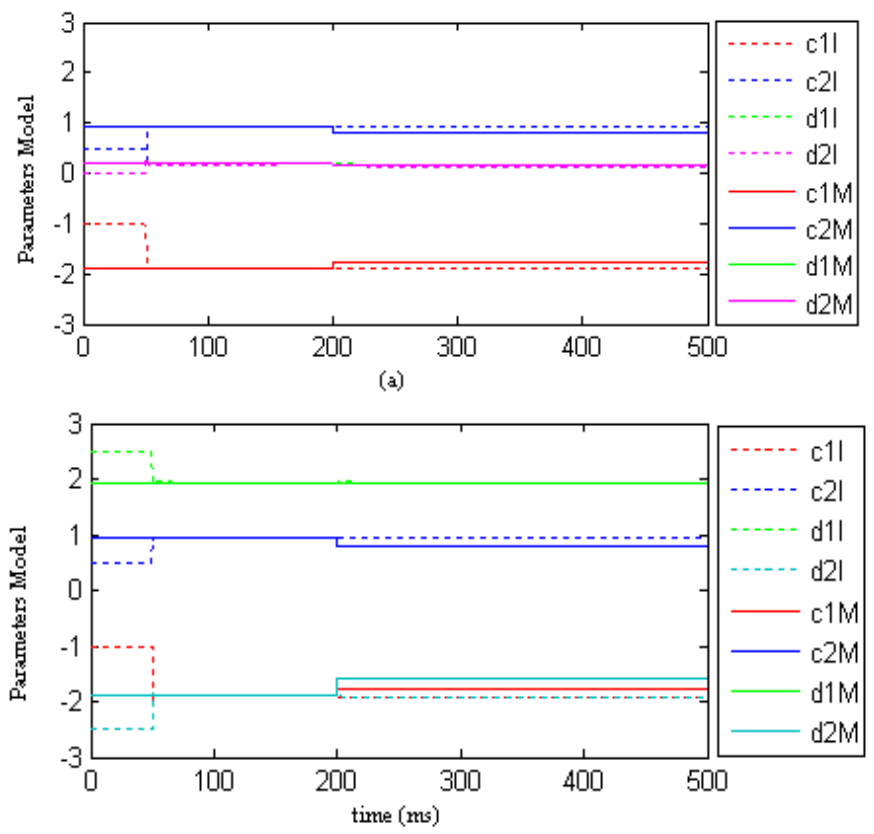

Fig.4: Parameters estimation for conventional RLS method at load change from 5-to-1 at $0.2 \mathrm{~s}$ (a) for voltage model, (b) for current model 
However, as shown in Fig.5, the effect of a step load change can be seen in the predication error of the voltage and current. Therefore, prediction error provides an opportunity to monitor the load change and may be considered in the identification algorithm. It is also important to note that during the load change there is a greater disturbance in the prediction error when using the voltage transfer function, rather than the current transfer function. Unlike the voltage transfer function model, the current transfer function model has a zero in the numerator. This gives a faster dynamic response due to pole canceling. For this reason, it is more difficult to use the current model to detect the load change using predication error.

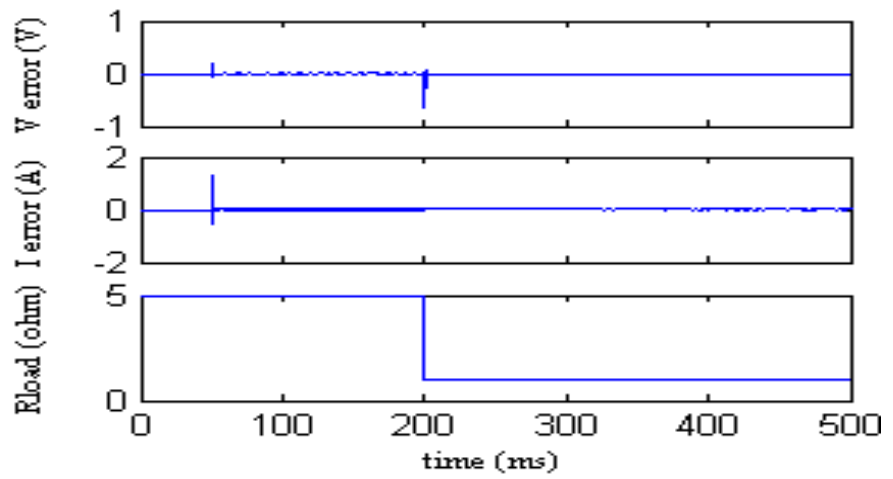

Fig.5: Prediction error for voltage and current at load change

The proposed method, shown in Fig.2, has been applied to monitor and estimate the load change. Again, a load change from 5-to- $1 \Omega$ is applied at $0.2 \mathrm{~s}$. The results from the voltage model are shown in Fig.6. The lumped parameters error at start up is less than $\pm 0.5 \%$ after ten iteration cycles $(2.5 \mathrm{~ms})$. After the load change, the parameter error is less than $\pm 2 \%$ for $\mathrm{c}_{1}$, and $\pm 1 \%$ for $\mathrm{c}_{2}$, with steady state error less than $\pm 2 \%$.

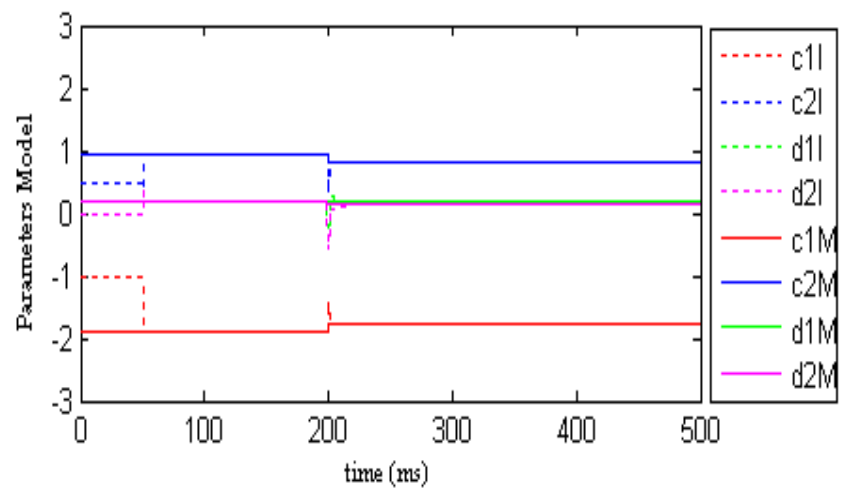

Fig.6: Parameters estimation for FRLS at load change from 5- to- $1 \Omega$

In practice, however, the most important parameter to update is the new value of load. Here, after the abrupt load changes to $1 \Omega$, the estimated load value is $1.0305 \Omega$ at ten iteration cycles. The rapid change in load estimation is shown in Fig.7.

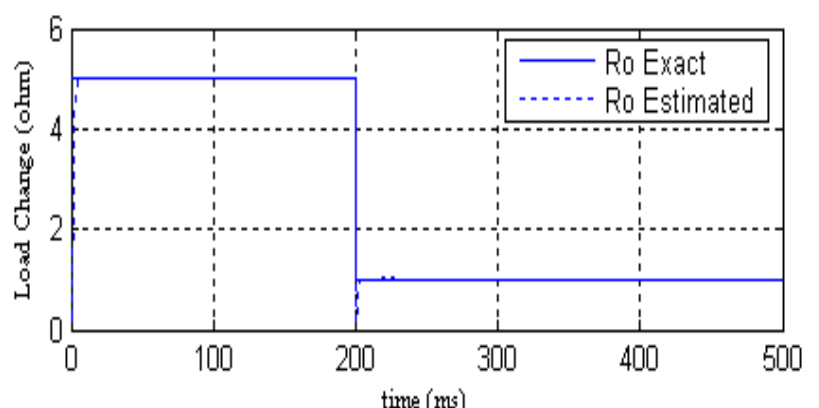

Fig.7: The comparison between estimation and exact load when the load changes from 5 -to- $1 \Omega$ at $0.2 \mathrm{~s}$

Fig. 8 shows the change of variable forgetting factor $(\lambda)$. This forgetting factor is directly linked to the parameter variation during the load change. The rapid change, and recovery, of the forgetting factor demonstrates the excellent ability of the method to track parameter changes.

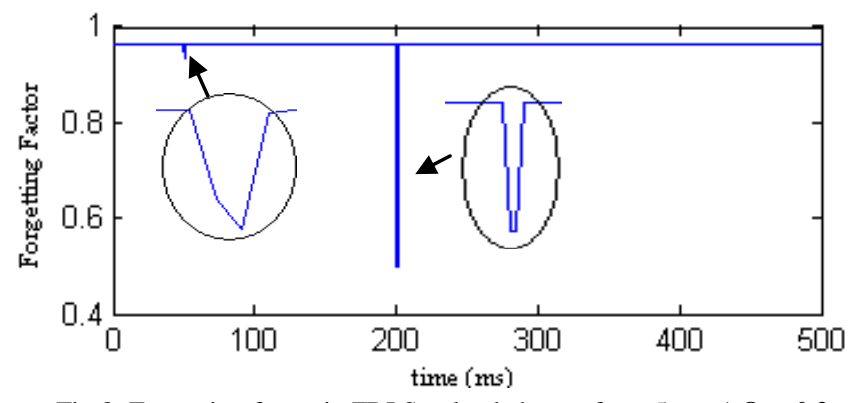

Fig.8: Forgetting factor in FRLS at load change from 5 -to- $1 \Omega$ at $0.2 \mathrm{~s}$

Further investigation into the proposed method has been carried out with a closed loop system using a conventional PI controller (Fig.9). Here, the control signal is injected by a PRBS with an 11 bit register and nominal signal amplitude of $\pm 0.01 \mathrm{~V}$. This results in a frequency rich signal (Fig.10). As shown in Fig.11, the algorithm successfully estimates the system parameters in a closed loop system very quickly and with accurate metrics. After the sudden change in load, convergence time is $2.5 \mathrm{~ms}$ and lumped parameter accuracy is less than $\pm 2 \%$.

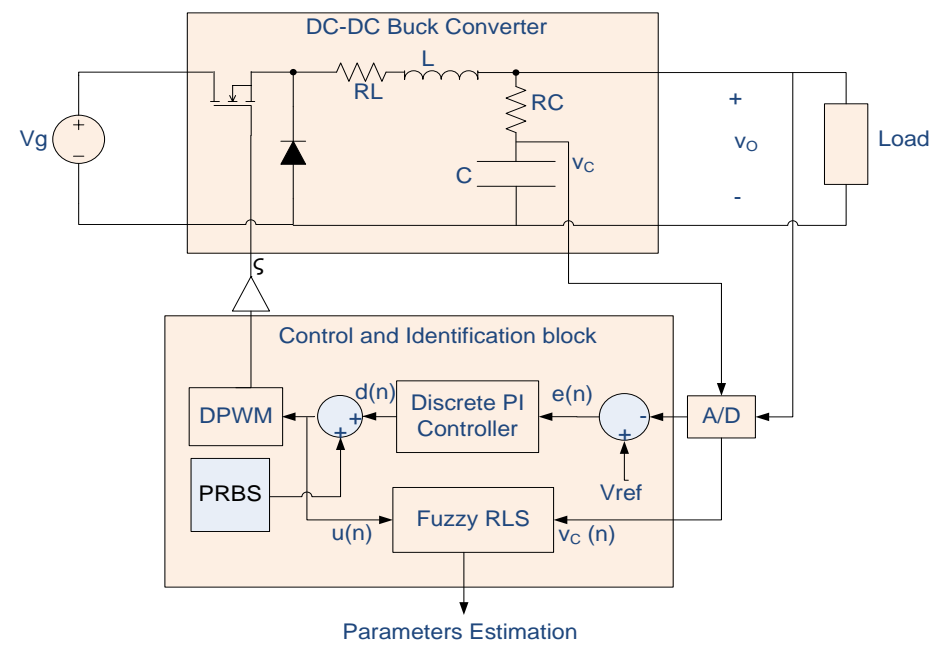

Fig.9: Practical block diagram of buck DC-DC converter with Fuzzy RLS identification module 

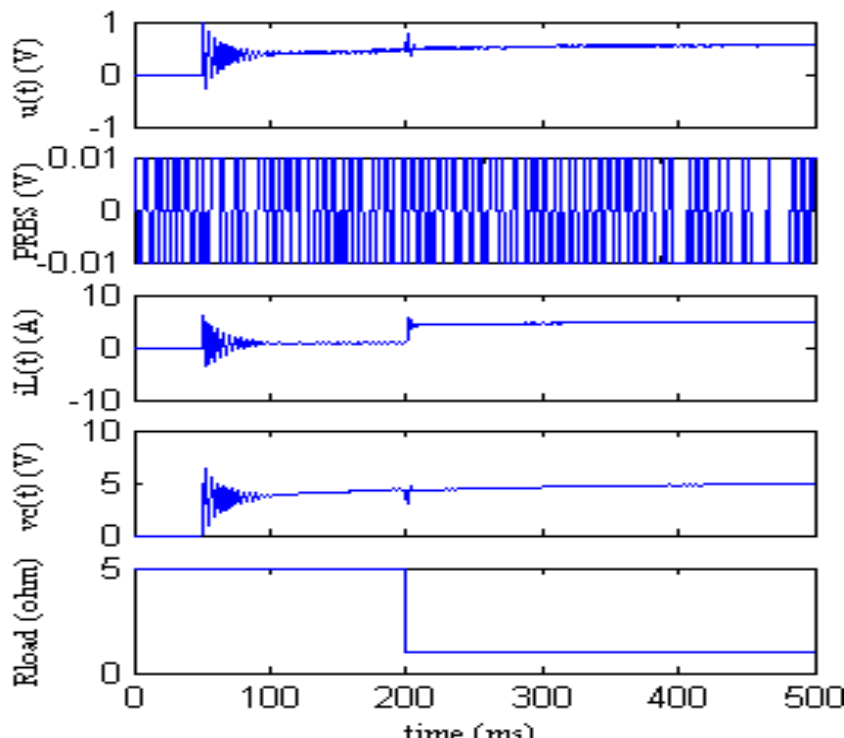

Fig.10: DC-DC digital signal at load change form 5- to- $1 \Omega$ at $0.2 \mathrm{~s}$

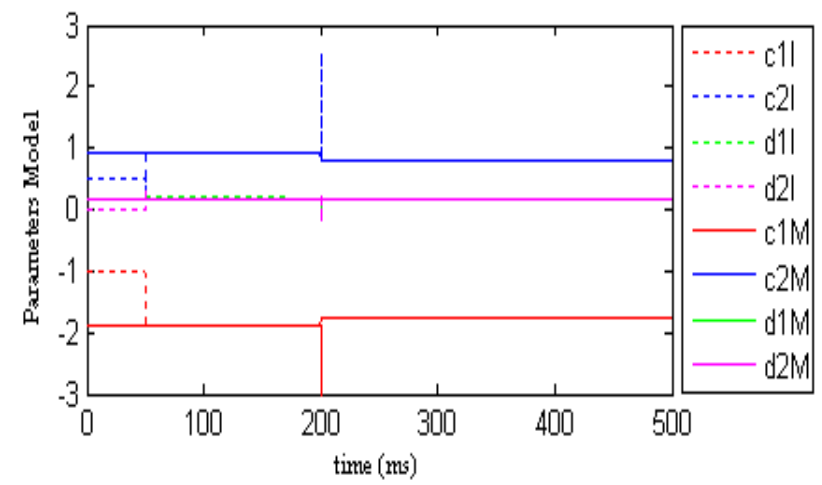

Fig.11: Parameters estimationfor FRLS, at load change in closed loop test

\section{Conclusion}

Parameter estimation has emerged as one of the most active research areas in the control of dc-dc converters. This paper has presented a two input, single output, fuzzy adaptive forgetting factor technique to improve parameters estimation during abrupt system load changes. The method has a simple structure, detecting a fast change in load via a sudden change in voltage prediction error. Results show that the convergence rate and parameter estimation are excellent in this method. An accurate model estimation can be achieved after ten iteration cycles, well before the output reach the peak value. Abrupt changes of load are adapted to very quickly and smoothly via the variable forgetting factor which simply responds to this load change. Results demonstrate that during fast load changes in dc-dc converters, estimation based on prediction error of the voltage model is preferred to the current model. Using the voltage model, a greater change in prediction error is observed during load change.

\section{ACKNOWLEDGEMENTS}

The authors wish to acknowledge Grant. E. Pitel, University of Illinois, for providing additional information relating to the work carried out in [8].

\section{REFERENCES}

[1] M. M. Peretz and S. Ben-Yaakov, "Time Domain Identification of PWM Converters for Digital Controllers Design," in Power Electronics Specialists Conference, 2007. IEEE. 2007, pp.809-813.

[2] Damian Giaouris, Soumitro Banerjee, Bashar Zahawi, and Volker Pickert: "Control of Fast Scale Bifurcations in Power Factor Correction Converter", IEEE Transactions of Circuits and Systems-II, vol. 54, no. 9, pp. $805-809$, Sept. 2007

[3] M. Shirazi, J. Morroni, A. Dolgov, R. Zane, and D. Maksimovic, "Integration of Frequency Response Measurement Capabilities in Digital Controllers for DC-DC Converters," Power Electronics, IEEE Transactions on power electronics, vol. 23, pp. 2524-2535, 2008.

[4] B. Johansson and M. Lenells, "Possibilities of obtaining small-signal models of DC-to-DC power converters by means of system identification," in Telecommunications Energy Conference, 2000. INTELEC. Twenty-second International, 2000, pp. 65-75.

[5] B. Miao, R. Zane, and D. Maksimovic, "System identification of power converters with digital control through cross-correlation methods," Power Electronics, IEEE Transactions on power electronics, vol. 20, pp. 1093-1099, 2005.

[6] Z. Zhao, "Design and Practical Implementation of Digital Auto-Tuning and Fast-Response Controllers for Low-Power Switch-Mode Power Supplies," in Electrical and Computer Engineering. Doctor of Philosophy ,University of Toronto, 2008, pp. 1-153.

[7] G. M. Buiatti, A. M. R. Amaral, and A. J. Marques Cardoso, "Parameter Estimation of a DC/DC Buck converter using a continuous time model," in Power Electronics and Applications, 2007 European Conference on, 2007, pp. 1-8.

[8] G. E. Pitel and P. T. Krein, "Real-time system identification for load monitoring and transient handling of Dc-Dc supplies," in Power Electronics Specialists Conference, 2008. PESC 2008. IEEE, 2008, pp. 3807-3813

[9] J. D. P. G.F.Frankline, and M.L.Workman,, Digital Control of Dynamic Systems, 3rd ed.: Menlo Park, Calif: Addison Wesley, 1998.

[10] J. B. V.Bobal, J.Fessl, and J.Machacek, Digital Self-tuning Controllers: Algoriths, Implementation and Applications: Springer-Verlag, 2005.

[11] W. Liang and R. Langari, "Identification of time-varying fuzzy systems," in NAFIPS/IFIS/NASA '94. Proceedings of the First International Joint Conference of the North American Fuzzy Information Processing Society Biannual Conference. The Industrial Fuzzy Control and Intelligent Systems Conference, and the NASA Joint Technolo, 1994, pp. 365-369.

[12] X. Yuncan, Y. Qiwen, and Q. Jixin, "Combined algorithm for systems with abrupt but infrequent parameter changes based on robust minmax and EW-RLS estimation," in Systems, Man and Cybernetics, 2003. IEEE International Conference on, 2003, pp. 177-180 vol.1. 